\title{
Tragedy of a Two-In-One Situation- A Rare Case Report on Gender Identity Disorder
}

\author{
Dr. Anindya Goswami ${ }^{1}$, Dr.Abhishek Das ${ }^{2}$, Dr. Sanchita Das Kundu ${ }^{3}$, \\ Dr. Saptarshi Chatterjee ${ }^{4}$, Dr. Tanmoy Sardar ${ }^{5}$, Dr. Sujash Biswas ${ }^{6}$ \\ ${ }^{1}$ Assistant Professor, Forensic \& State Medicine, Nil Ratan Sircar Medical College, Kolkata \\ ${ }^{2}$ Assistant Professor, Department of Forensic Medicine \& Toxicology, Sikkim Manipal Institute \\ of Medical Sciences, Gangtok, Sikkim, India \\ ${ }^{3}$ Assistant Professor, Forensic \& State Medicine, Bankura Sammilani Medical College \\ ${ }^{4}$ Demonstrator, Forensic \& State Medicine, Nil Ratan Sircar Medical College, Kolkata \\ ${ }^{5}$ Demonstrator, Upgraded Department of Forensic \& State Medicine, Medical College, Kolkata
}

\begin{abstract}
With the advancing civilization, the complexity of human mind is also rising. It brings psychological disturbances and upsets off and on. The proper upbringing of a child plays vital role in psychiatric development of an individual in later life. Here, the gender identity takes pivotal role, in disturbance of which there come numerous psychiatric disorders and adverse situations. Many a times the person affected becomes unable to combat the stress created by the gender identity disorder. Gender identity disorder is said to be one of the most suppressed psychiatric entity. Studies show that there is a very close relationship with depression, which often leads to suicidal tendency. This is a rare case report where a person, after going through "sex-change intervention" by removal of the penis, scrotum and testes at an early age, suffering from depression resulting from gender identity disorder, died of suicidal hanging. This incidence proves the necessity of dealing these situations with utmost importance and care.
\end{abstract}

Key words: Psychiatric development, Gender identity disorder, Depression, Sex-change intervention, Suicidal hanging

\section{Introduction}

Gender Identity Disorder (GID) is a complex disorder marked by persistent discomfort about once assigned sex, discontent with once designated birth sex, a desire to have the characters of the other sex and to be regarded socially as a person of opposite sex. GID is associated with significant impairment in social, occupational, interpersonal and the other areas of functioning. ${ }^{[1]}$

It is one of the most controversial diagnoses of DSM-IV and almost incomparable in the complexity of its social, ethical and political considerations to any other diagnosis. Careful history taking reveals underlying conflicts with prominent gender dysphoria and social complexities. ${ }^{[2]}$

Moreover, transgender population is not a rarity in Indian perspective and due to economical pressure of family. Often they are forcefully made "hijra" or "zenana" for the livelihood, which creates immense social and psychological pressure.

It is unfortunate that most local media depict transsexuals in a negative light. Public view upsets the psychological and emotional state of a transgender, given that most face an unsympathetic atmosphere and consider them as deviant in cultures that are unable to play a constructive role. ${ }^{[3]}$ The aetiology is multi-factorial and still demands clear explanation.

The present case is also of such a poor person who was made female from male in childhood. Gradually he succumbed to psychological jeopardy and scarcity of livelihood.

\section{Case History}

The subject used to stay at a suburban region near Kolkata and was known to the locality by a unisexual name (a name that can be addressed to both male and female). He resided separately from his parents in a rented room in the same locality. He was emasculated by removal of scrotum, testes and penis at an early age and was eunuch (hijra) by behaviour and profession. On the day of incidence, the mother of the subject went to call the person in the evening when she found the room to be locked from inside. Upon opening, the subject was found hanging from the ceiling by a scarf around his neck with foot touching the ground. When removed immediately to nearest Govt. hospital, he was declared brought dead. 


\section{Autopsy Findings}

Suspicious and unnatural death, medicolegal autopsy was done on the next morning. It was a body of a male subject phenotypically having rigor mortis all over the body; both eyes closed with hazy cornea and dilated fixed pupils. Scalp hair was 14" black and there was evidence of dried salivary stain over right angle of mouth and chin. Over the region of external genitalia, there was evidence of one longitudinal old surgical scar mark with removal of testes, scrotum and penis with only presence of a urethral opening. One non-continuous ligature mark 13" x 2" placed obliquely high up around neck with gap of 2.5 " over left mastoid region. The upper margin of the ligature mark present 1 " below left angle of mandible, 2.5 " below chin, 1.5 " below right angle of mandible \& 1" below tip of right mastoid process. Skin on the ligature mark was brownish, parchmentised, furrowed and abraded at places. On dissection, the subcutaneous tissue under the ligature mark was whitish, hardened, condensed and glistening in appearance without any extravasation of blood in and around. Injuries showed evidence of vital reactions. Mucus membrane of larynx and trachea were congested, but there was no fracture of hyoid bone and thyroid cartilage. Pleura were congested with petechial hemorrhagic spots on visceral surface. All the other organs including lungs were congested. It was in fact a case of death due to antemortem suicidal partial hanging.

\section{Discussion}

In our country, hijras and zenanas are neglected part of the society. They earn their livelihood by treating and dancing newborns or other minor ways. No doubt it is tough fight for life of them socially, psychologically and economically as establishment and maintenance of gender secrecy can have psychosocial consequences and other deleterious effects. This subject was no exception. Further police enquiry into this case revealed that he was frustrated recently to earn his livelihood and often found depressed and socializing with others. This is a case of a complex tragedy where the subject was attempted to change the gender identity to that of a female at early age. It is obvious that senses of unbearable identity confusion lead to the depression in his mind as he could not decide which life to be lived- a male or a female? And the ultimate result was the suicidal attempt in the background of transgender identity. ${ }^{[4]}$

Before the 1960s, secrecy and confusion dominated the area of atypical gender identity development. The first definition of the term 'gender role' was given by John Money (1955). Money wanted to differentiate a set of feelings, assertions and behaviours that identified a person as being a boy or a girl, or a man or a woman, from the contrasting conclusion one could have reached by considering only their gonads. In the vast majority of cases he described, the gender role adopted by the individuals was consistent with their rearing. The term 'gender identity' appeared in the mid-1960s in association with the establishment of a gender identity study group at the University of California. Stoller defines it as: "A complex system of beliefs about oneself: a sense of one' masculinity and femininity. ${ }^{[4]}$

Gender Identity Disorder is not an uncommon problem all over the world. These people again and again exhibit a desire to express themselves in a way that is not socially acceptable for their assigned sex from an early age. ${ }^{[3]}$

Children, particularly adolescents, and their families often find the experience of a gender identity disorder painful and unbearable, and adolescents are at high risk of suicide attempts. This sense of despair frequently leads to extreme pressure being placed on clinicians to act and to provide immediate solutions, through physical intervention, which may not be clinically appropriate at the time. ${ }^{[4]}$

Studies have shown these people to present with separation anxiety, depression and emotional and behavioural difficulties. In a small percentage of cases, child sexual abuse has been associated with a gender identity disorder. Suicide attempts in adolescence or young adulthood are frequent and in some cases this is how cases come to professional attention. ${ }^{[4]}$

Gender differences have been reported in two neurotransmitter systems traditionally implicated in the Pathophysiology of depression, namely noradrenalin and serotonin. Hormonal influences may have a role. ${ }^{[5]}$

\section{Conclusion}

The concept of gender identity and role having been formulated, it became possible to make sense of, and give order to, conditions that had been ill-defined and poorly understood earlier. Incongruity between the biological sex and the psychological behavioural manifestations of gender identity is indicated in these cases. The incidence of childhood cross-gender identification in the general population and in the psychiatric population has not yet been definitely established. No large-scale investigation with standardised criteria, such as those of DSM-IV, has yet been conducted. No single cause has yet been found with certainty for the development of a gender identity disorder.

Hereditary factors have been suggested for male homosexuals. The contribution of factors to the development of it is unclear and further research is needed. A parent's wish for a child of the other gender, or 
direct parental pressure in rearing the child in the gender opposite to the biological sex is prominent in most cases.

A number of authors agree that many of these factors present simultaneously and work together during a critical period to produce depression in trans-genders. This would also explain the rarity of the condition.

Behavioural therapy, individual psychotherapy, family therapy and group therapy are needed with these subjects and their families. ${ }^{[4]}$ Management by pharmacotherapy and harm reduction model have been beneficial in many cases. ${ }^{[2]}$ Lifetime psychiatric co-morbidity is high, and this should be taken into account in the assessment and treatment planning. ${ }^{[6]}$

Early identification and timely intervention may lead to the remission by saving the life of these patients.

Conflict Of Interest: This article was not sponsored by anyone and was done exclusively by the authors with their own resource and interest.

\section{Source of Funding: Nil}

Ethical Clearance: Not applicable

\section{Reference}

[1]. Arya A, Tripathi A, Singh H, Kumar P. Gender Identity Disorder With Co-morbid Depression -A Case Report.Delhi Psychiatry Journal. 2010 Apr; 13(1):153-156

[2]. Kumar K, Gupta M. Social dichotomy versus gender dichotomy: a case report of gender identity disorder. Indian J Psychol Med. 2012 Apr;34(2):190-192

[3]. Syed MA, Abdul GK. Gender Identity Disorder is Not Simply Two in One. The International Medical Journal Malaysia.2013 Dec; 12(2): 83-85

[4]. Cegile DD.Gender identity disorder in young people. Advances in Psychiatric Treatment. 2000; 6: 458-466

[5]. Piccinelli M, Wilkinson G. Gender differences in depression. British journal of Psychiatry. 2000; 177: 486-492

[6]. Hepp U, Kraemer B, Schnyder U, Miller N, Delsignore A. Psychiatric co-morbidity in gender identity disorder.Journal of Psychosomatic Research. 2005 Mar; 58(3): 259-261

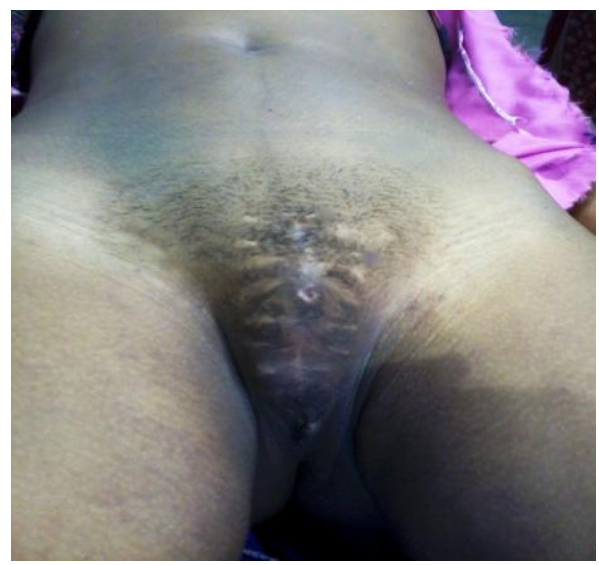

Fig.1- The abdomen and external genitalia (Note the prominent feminine features)

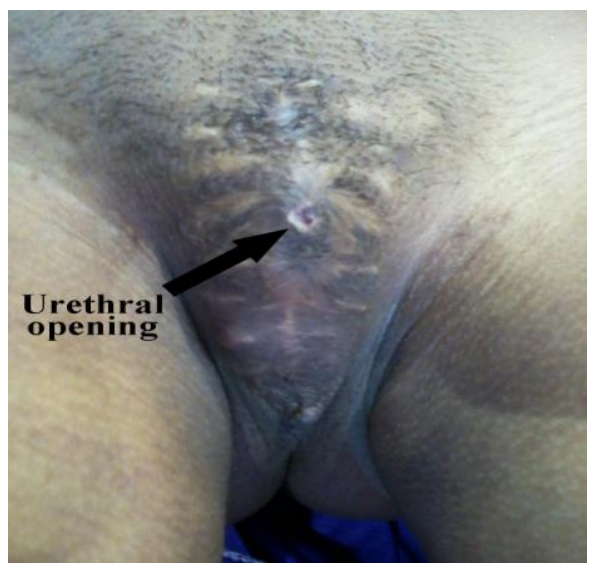

Fig.2- A closer look of genital region (The arrow points the urethral opening) 